\title{
Molecular evolution analysis of the antioxidant system and heme metabolism in helminths
}

\author{
D. Konstantinov ${ }^{1,2 *}$, M. Lvova 2 , A. Doroshkov² \\ ${ }^{1}$ Novosibirsk State University, Novosibirsk, Russia \\ ${ }^{2}$ Institute of Cytology and Genetics SB RAS, Novosibirsk, Russia \\ *e-mail: konstantinov@bionet.nsc.ru
}

Key words: heme, helminths, evolution, antioxidant system

Motivation and Aim: Often endoparasite feed on blood and harmful to vertebral (schistosomiasis, opisthorchiasis, etc.). Common adaptations to parasitism are the reduction of morphology and the change in biochemistry. For the normal functioning of the parasite-hematophagus the most important are the heme metabolism system and the antioxidant system. The choice of these two systems is due to the fact that with this type of food, a large number of blood components enter the parasite organism and it needs to utilize toxic excess iron and heme derivatives. Also the activity of immune cells (they produce reactive oxygene species), and chemical conditions ( $\mathrm{pH}$, etc.). These enzymatic systems of many parasites may be targets for therapy. The automatic annotation is complicated due to the large evolutionary distances between model organisms (such as $C$. elegance and $S$. mediterranea) and the most important human hematophagous helminths (schistosomes, opisthores, etc.). In our report, we present a comprehensive study of the molecular evolution of genes encoding enzymes of biosynthesis and heme degradation, as well as antioxidant systems of hematophagous helminths.

Methods and Algorithms: Using the text mining technology we find targets of drags in a model organism (such as $S$. mansoni, O. viverrini, H. robusta, N. americanus). For each target was performed extraction of sets of homological sequences presets from databases was carried out using the reciprocal BLAST search. For each sample set was conducted a domain compositions and protein secondary structure comparative analysis. During molecular phylogeny reconstruction orthologues and paralogues relationships, as well as the functional correspondence between proteins were identified.

Results: Complete set of heme biosynthesis genes identified in Flatworms, this is probably related to the fact that part of the lifecycle of the species conducts in the external environment. At the same time a number of differences in protein structure were revealed. Differences in the set of genes responsible for the synthesis of components of antioxidant systems, as well as genes of enzymes responsible for heme degradation, have been revealed, indicating significant differences in the organization of these systems between free-living and parasitic organisms. The obtained data allows us to define targets for further development of modern methods of control of helminths.

Conclusion: A number of differences in protein structure were revealed, for example, the difference in the domain composition of proteins catalyzing the reaction of the formation of 5-aminolevulinic acid from glycine and succinyl-CoA suggests a different protein localization in the cell. 